\title{
The dynamics, kinetics and reversibility of protein adsorption onto the surface of biodegradable materials
}

\author{
Catarina M. Alves, ${ }^{* a b}$ Rui L. Reis ${ }^{a b}$ and John A. Hunt ${ }^{c}$ \\ Received 11th February 2010, Accepted 10th May 2010 \\ DOI: 10.1039/c002972f
}

Dynamic contact angle (DCA) analysis was used to investigate the kinetics and dynamics of protein interactions in a time-dependent manner for a group of organic natural-based surfaces during their initial contact with aqueous and protein solutions. Starch-based biomaterials were used to analyze the influence different materials with different surfaces had on the adsorption, desorption and configuration of proteins. Polymeric blends of starch and cellulose acetate (SCA), polycaprolactone (SPCL) and ethylene vinyl alcohol (SEVA-C) were used. The model protein systems included single protein solutions of human serum albumin, fibronectin, vitronectin and fibrinogen, and also complex solutions of human blood plasma. In the adsorption studies, very small and nearly equal advancing and receding contact angles were measured for all the materials. Highly wetting and low contact angle hysteresis, therefore, are exhibited by these surfaces. This effect was more noticeable for SCA surfaces. Moreover, the effect of protein concentration was also assessed and demonstrated to substantially affect the DCA wetting forces of SEVA-C and SPCL surfaces. In the desorption studies, during the rinsing phase with saline solution, the DCA loops became larger than that observed for the adsorption phase, which indicated increases in the contact angle hysteresis. The hysteresis of SCA and SPCL surfaces reversibly changed through the desorption phase, at the end of which, hysteresis was comparable to that of surfaces immersed in saline solution. The results indicated that adsorbed proteins could desorb more readily on SCA and SPCL than on SEVA-C. In the later case, stronger interactions such as hydrophobic forces were established and it is likely the rearrangement of protein conformation had occurred. Monitored by DCA, the evolution of hysteresis demonstrated the progressive bond strengthening between protein molecules and solid substratum, further elucidating the behaviour of proteins that regulate cellular interactions with implanted devices.

\section{Introduction}

The paradigm of cell material interactions, which proposes that protein adsorption is the first event following direct contact and this itself determines the cascade of subsequent interactions of cells, could be central to the design of new strategies for biocompatibility ${ }^{1}$ and tissue engineering. ${ }^{2,3}$ From a functional point of view, it is accepted that qualitative and quantitative assessment of the affinity of proteins to surfaces is essential to evaluate cell mechanisms upon attachment to the surfaces and thus develop improvements in the properties of implanted materials. Proteins such as fibronectin and vitronectin are known to interact with biomaterial surfaces shortly after implantation. Plasma proteins are immediately adsorbed onto the surface of biomaterials and are known to determine subsequent cell related events. ${ }^{4}$ Protein adsorption events have a central importance in the regulation of initial cell mechanisms such as adhesion that in turn will modulate critical later stage biological processes. ${ }^{5,6}$

a3B's Research Group - Biomaterials, Biodegradables and Biomimetics, University of Minho, Headquarters of the European Institute of Excellence on Tissue Engineering and Regenerative Medicine, AvePark, 4806-909 Taipas, Guimarães, Portugal

${ }^{b} I B B$ - Institute for Biotechnology and Bioengineering, PT Government Associated Laboratory, Guimarães, Portugal

${ }^{c}$ Clinical Engineering, UKCTE, School of Clinical Sciences, University of Liverpool, P.O. Box 147, Liverpool, L69 3QA, United Kingdom
Notwithstanding the relevance of adsorption, in the field of surface science, increasing relevance has been attributed to the study of adsorption-desorption kinetics or adsorption reversibility related to folding-unfolding events. Different substrates interact differently with these molecules resulting in conformational changes of the protein structures, thus defining the binding quality of specific cell receptors. ${ }^{7}$

Several methodologies are available for the study of protein interactions with macromolecules providing information on the amount of protein or molecular conformation. ${ }^{8-10}$ Yet, proteinpolymer interactions are not directly evaluated. ${ }^{11}$ Moreover, proteins are essentially large hydrocarbons with more complex structures and as such their identification is highly complex. Detection problems are even more apparent at submonolayer coverage, where the substrate adds greater complexity to the analytical spectrum. Proteins are difficult to distinguish from "contamination" by other hydrocarbon species that can be present on surfaces, such as starch based ones; also the intensity of spectral features that can be used for identification is often so weak that detection limits are low and sensitivity is poor. ${ }^{11}$ Useful information could be provided by the study of the dynamics of interfacial reactions such as those triggered by the contact of a material's surface with a biological medium.

Although the adsorptive characteristics of a surface are determined by its wetting tension and wettability, ${ }^{12}$ the real time dynamics of these parameters in protein adsorption have hardly 
been investigated. Dynamic Contact Angle (DCA) analysis, developed by Andrade et al. ${ }^{4}$ has proven to be a useful technique for a first-order interpretation of these dynamic interfacial interactions. DCA is sensitive to the outermost few angstroms of the surface and thus provides a powerful means of monitoring submonolayer changes of the substrate., ${ }^{4,13}$ Currently, the tensiometric DCA has proven to be extremely useful for biomaterial characterization. ${ }^{14,15}$ This technique is generally used to measure the advancing and receding contact angle of water on material surfaces and can therefore be used to determine surface changes by the measurement of the variation in the contact angles (hysteresis). From a thermodynamic perspective, advancing and receding contact angles should be equal. Yet, in experimental systems hysteresis is detected and two types are generally considered: thermodynamic and kinetic hysteresis. ${ }^{4,16,17}$ DCA provides information from the material side that allows the detection of quick changes in the surface configuration. ${ }^{18}$ The method yields data on a multitude of surface characteristics, such as the presence of chemical and physical heterogeneities, changes in the surface configuration and adsorption-desorption processes. DCA allows the study of sample surfaces while in contact with the biological model fluid, providing a continuous, non-destructive monitoring technique ${ }^{19}$ that detects changes over increasing time. ${ }^{4}$ The formation of a biofilm from a protein solution also takes place on the same timescale. ${ }^{20}$ In protein adsorption studies, changes in DCA hysteresis can reflect the adsorption of proteins onto surfaces forming a biofilm. Upon adsorption, proteins may undergo conformational changes that allow hydrophobic residues to contact a hydrophobic surface, exposing hydrophilic residues towards the solution. This results in stronger bonds between proteins and surfaces and can lead to a more uniform and hydrophilic surface chemistry, ${ }^{21}$ that in turn can alter further cellular reactions.

Starch-based biomaterials (SBB) have been increasingly studied for applications in the field of Tissue Engineering, ${ }^{22,23}$ including their use as scaffolds for bone related applications ${ }^{24}$ and drug delivery systems. ${ }^{25}$ Based on these advantages and on the already reported immunological response to implanted starch based materials, ${ }^{26}$ this study was conducted to evaluate the dynamics of protein interaction on the different surfaces. The work disclosed herein focused on obtaining the DCAs of different surfaces for single solutions of human proteins: serum albumin (HSA), fibronectin (HFn), vitronectin (HVn), fibrinogen (HFbg); and for complex solutions such as human blood plasma. The DCA technique was used to investigate (1) the adsorption of macromolecules, (2) protein concentration effects, (3) the adsorption and desorption rates and (4) partial denaturation effects driven by the surface characteristics. Moreover, visualization of the final surfaces was analysed by Laser Scanning Confocal Microscopy (LSCM).

\section{Materials and methods}

\subsection{Starch-based biomaterials (SBB)}

Different biodegradable polymeric blends of corn starch with: (i) cellulose acetate (SCA), (ii) ethylene vinyl alcohol copolymer (SEVA-C) and (iii) polycaprolactone (SPCL) were studied. The amount of starch was $50 \%$ by weight (wt $\%$ ) in SCA and SEVA-C and $30 \%$ wt. in SPCL. By means of using conventional injection moulding technology, samples were processed into $10 \mathrm{~mm}$ circular discs. Samples were sterilized by ethylene oxide under optimised conditions, ${ }^{27}$ washed, and subsequent experimental procedures were performed under clean conditions.

\subsection{Proteins and human blood plasma collection}

Dynamic contact angle studies were performed using different commercially available human origin biomolecules: HSA, HFn, HVn and HFbg (Sigma-Aldrich, UK). Protein solutions were prepared in Phosphate Buffered Saline (PBS; Sigma-Aldrich, UK) solution ( $\mathrm{pH}$ 7.4). To assess the effect of protein concentration in DCA hysteresis, $35 \mu \mathrm{g} \mathrm{mL}^{-1}$ and $70 \mu \mathrm{g} \mathrm{mL}^{-1} \mathrm{HSA}$ solutions were prepared, which corresponded to $0.1 \%$ and $0.2 \%$ of its physiological concentration. Concentrations of $\mathrm{HFn}, \mathrm{HVn}$ and $\mathrm{HFbg}$ solutions were prepared at $0.2 \%$ of their amount in the human blood: $0.8 \mu \mathrm{g} \mathrm{mL}^{-1}, 0.6 \mu \mathrm{g} \mathrm{mL}^{-1}$ and $0.4 \mu \mathrm{g} \mathrm{mL}^{-1}$, respectively. Furthermore, to analyse the effect of complex protein solutions on DCA hysteresis, human blood plasma was used. In brief, whole blood was collected from healthy unmedicated, adult volunteers, anticoagulated with $0.002 \%$ of heparin and centrifuged at $2500 \mathrm{rpm}$ for $5 \mathrm{~min}$ at $4{ }^{\circ} \mathrm{C}$. Human blood plasma solutions were prepared at $0.2 \%(\mathrm{v} / \mathrm{v})$ in PBS solution.

\subsection{Dynamic contact angle: protein adsorption and desorption}

2.3.1. Theoretical principles. DCA measurements were performed based on the Wilhelmy plate method. ${ }^{28}$ Wilhelmybalance tensiometry was performed using a computer-controlled instrument (Camtel CDCA 100F, Royston, UK). The theoretical principles of this methodology are described in the literature. ${ }^{11,17}$ Briefly, a plate was immersed and emersed from the model liquid and the forces acting in the specimen were recorded by electrobalance. According to the procedure, the balance was reset to zero and linear regression was performed to the immersion depth zero. This provided for the elimination of the weight of the sample and buoyancy forces. The relation between force and surface tension was represented by the following equation:

$$
\frac{F}{L}=\gamma_{10} \cos \theta
$$

where $F$ is the force in $\mathrm{mN}, \gamma_{10}$ the surface tension of the liquid and $\theta$ is either the advancing $\left(\theta_{\text {adv }}\right)$ or receding $\left(\theta_{\text {rec }}\right)$ contact angle.

Finally, contact angle hysteresis is the difference between advancing and receding contact angles. This parameter is affected by the distribution of different chemistries on the surface with different properties and thus is a measure of the homogeneity of the surface.

2.3.2. Adsorption and desorption studies. The DCA studies were performed running multiloop DCA analysis at controlled temperature $\left(22^{\circ} \mathrm{C}\right)$. For the experimental set up, all loops were measured at wetting (immersion) and dewetting (emersion) rates $60 \mu \mathrm{m} \mathrm{s}^{-1}$ and the immersion depth was $2 \mathrm{~mm}$. In all experiments, a single hysteresis loop lasted $3 \mathrm{~min}$ and therefore a complete 60 -loop experiment ran for approximately $3 \mathrm{~h}$. The duration of the experiments and subsequently the number of DCA cycles was 
selected based on previous studies with starch-based biomaterials showing that HSA and HFn adsorption was completed in approximately $90 \mathrm{~min}$ after incubation. ${ }^{29}$

Prior to the evaluation of the effect of the different protein conditions on the advancing (adv) and receding (rec) dynamic contact angles (DCAs), ultra-pure water and PBS solution were studied. The fitting of the advancing arm, the transition from advancing to receding mode and the receding arm were determined; finally, hysteresis $(H)$ was calculated. The effect of the different proteins and human blood plasma on the advDCAs and recDCAs of SCA, SEVA-C and SPCL was investigated. In the adsorption study, experiments were performed using each protein solution for 60-loops. In contrast 30-loop desorption studies were performed in protein free buffer (desorption phase) directly after 30-loop experiments of the adsorption phase.

\subsection{Protein labelling}

Antibody labelling of specific biological molecules was selected to analyse the adsorption of albumin, fibronectin, vitronectin and fibrinogen on the different surfaces. For the detection of the biomolecules, samples used in the adsorption and desorption DCA studies were fixed using 4\% formaldehyde solution and washed with PBS solution. Initially, the materials were exposed to horse serum for $20 \mathrm{~min}$, followed by incubation with primary antibodies for $30 \mathrm{~min}$ at $37{ }^{\circ} \mathrm{C}$. For the identification of the different biomolecules, the following antibodies were used: sheep anti-Human Albumin, Fibronectin, Vitronectin, and Fibrinogen (Farnell, UK). After the primary antibody, materials were incubated with donkey anti-sheep Alexa Fluor 488 antibody (Molecular Probes, The Netherlands) for $1 \mathrm{~h}$ at $37^{\circ} \mathrm{C}$.

\subsection{Confocal laser scanning microscopy}

Samples obtained after adsorption and desorption experiments were analysed by Confocal Laser Scanning Microscope (LSM 510 Zeiss, UK) using an argon laser $(\lambda=488 \mathrm{~nm})$ for the excitation of the probe-conjugated antibodies used to detect adsorbed HSA, HFn, HVn and HFbg. Image analysis was performed using KS400 image analysis software (Imaging Associates, UK).

\section{Results and discussion}

\subsection{Dynamic wettability measurements}

The dynamics of the advancing and receding wetting tension were detected by means of force loops. Dynamic ten-loop experiments in PBS determined a fundamental difference between the different starch based materials studied. Fig. 1 shows the plots of force versus immersion depth for the three materials. The lower horizontal portion of the loop is the immersion (measuring the advancing angle) and the upper portion is the withdrawal (measuring the receding angle) of the sample.

The force loops using ultra-pure water and PBS were similar although a slight decrease in hysteresis was observed due to the influence of the phosphate buffer in the homogenization of the surfaces. For each cycle, the DCA loops for the surfaces of SEVA-C and SPCL were similar (Fig. 1b and 1c) with no reduction in hysteresis after the initial change from dry to wetted state from the second loop on. In contrast, SCA surfaces showed a time-dependent wetting behaviour. Indicated by the immersion loops, with increasing loop number and time of surface/PBS contact, the area of the SCA material that becomes totally wettable increases. The lowest hysteresis was obtained at loop 10 at which the steady state was achieved (Fig. 1a).

In theory, the occurrence of contact angle hysteresis is not predicted for "ideal" surfaces. ${ }^{4}$ Yet, surfaces generally present either time invariant hysteresis (thermodynamic or true hysteresis) or time-dependent hysteresis that results from the re-equilibration phenomenon taking place in the surface-liquid interface (kinetic hysteresis). ${ }^{4,16,17}$ DCA analysis of hysteresis proved to be a sensitive method to assess surface configuration changes. The results demonstrated examples of kinetic (Fig. 1a) and thermodynamic hysteresis (Fig. 1b and 1c). The increase in hydrophilicity was the fundamental difference between SCA, SEVA-C and SPCL polymeric blends. SCA changed completely into a wettable surface at loop 10 after the initial hydrophobic loops. Time-dependent contact angle hysteresis resulted from the changes of the advancing angles, as the variation of receding contact angles was very small.

According to the literature, ${ }^{17,21}$ the kinetic hysteresis observed for SCA could be due to swelling, surface mobility or to changes in surface configuration. In opposition, the hysteresis observed for SEVA-C and SPCL could be caused by surface roughness and chemical inhomogeneity, absent in the so called ideal surfaces. ${ }^{17}$ The results can be further interpreted by considering the miscibility and interaction between the synthetic and natural phases of the materials. Previous studies reported the heterogeneity of SCA surfaces in opposition to the higher homogeneity of SPCL and SEVA-C. ${ }^{30,31}$ The more homogeneous a material is the lower the degree of freedom at the molecular level and thus changes in the surface configuration are limited. This could explain the invariant thermodynamic hysteresis observed for
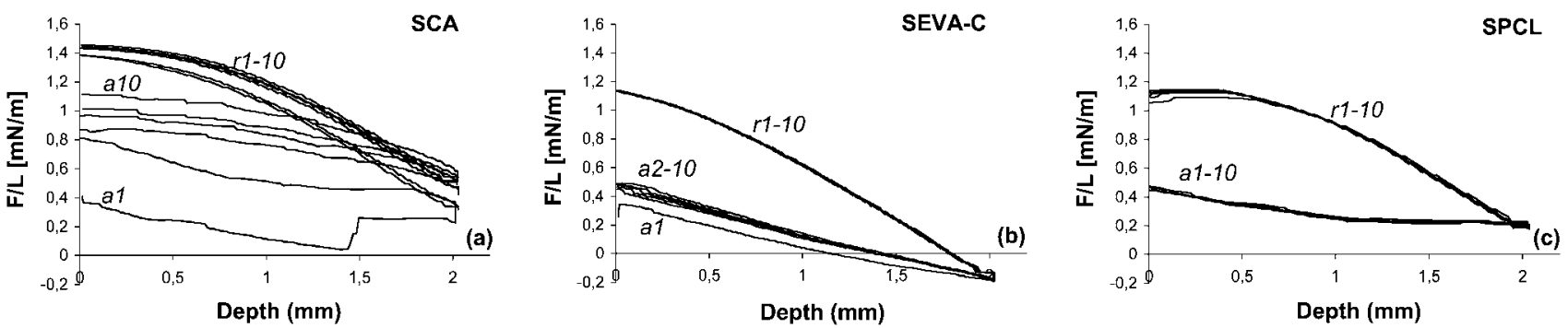

Fig. 1 DCA loops of SCA (A), SEVA-C (B) and SPCL (C) immersed in PBS solution. The advancing $(a)$ and receding $(r)$ loop numbers (1-5 and 10) were indicated. 

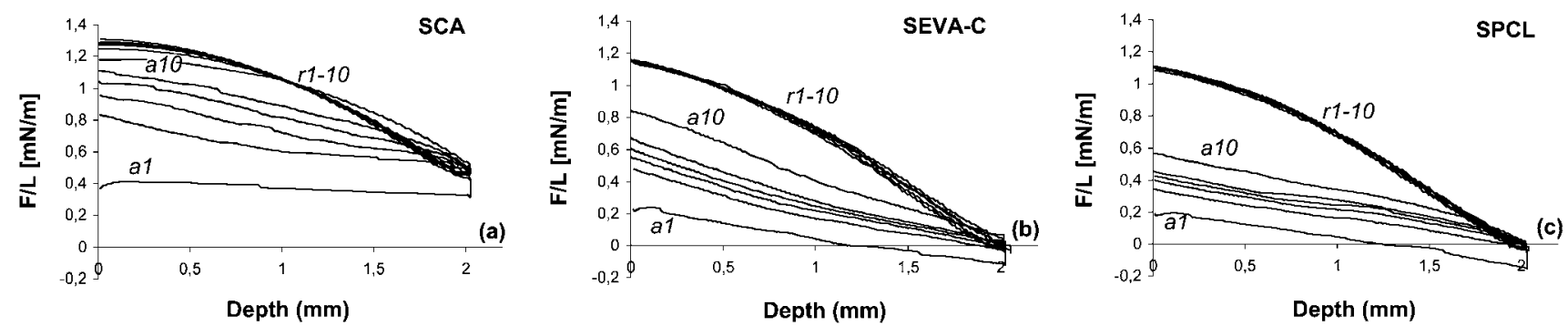

Fig. 2 DCA loops of SCA (A), SEVA-C (B) and SPCL (C) immersed in a solution of $70 \mu \mathrm{g} \mathrm{mL} \mathrm{L}^{-1}$ of HSA (adsorption phase). The advancing (a) and receding $(r)$ loop numbers (1-5 and 10) were indicated.

SEVA-C and SPCL. An empirical model to explain the correlation between surface homogeneity and contact angles was derived by Johnson and Detre. ${ }^{32,33}$ According to this model, advancing DCAs of the macroscopically heterogeneous surfaces were influenced by $10 \%$ hydrophobic surface patches, while $10 \%$ hydrophilic areas in a hydrophobic surface affected the receding DCAs. On the heterogeneous SCA surfaces time-dependent hysteresis resulted from changes of the advDCAs. Developing from this, it could be stated that SCA presents hydrophobic patches that represent over $10 \%$ of the surface.

\subsection{Protein adsorption analysis: effect of solution concentration}

The 10-loop DCAs in $70 \mu \mathrm{g} \mathrm{mL} \mathrm{mL}^{-1}$ HSA (Fig. 2) indicated differences between the advancing angles for SCA, SEVA-C and SPCL while, in all cases, receding angles remained constant. The shape of the DCA curves observed for all surfaces substantially changed when compared to the protein-free solution (Fig. 1). This variation was slower for SEVA-C and SPCL. This indicates a delay in hydrophilization when compared to the faster hysteresis obtained for the SCA surface.

To analyse the effect of protein concentration on hysteresis, the data from the 30 loop experiments were presented as a function of time or cycle number (Fig. 3). Albumin solutions of $35 \mu \mathrm{g}$ $\mathrm{mL}^{-1}$ and $70 \mu \mathrm{gL}^{-1}$ were used as test solutions. For all materials, the data showed that an increase in solution concentration resulted in a decreased hysteresis. These results were in agreement with other studies ${ }^{19,21}$ that indicated the reduction of hysteresis with increasing concentration of bovine serum albumin (BSA) solutions. The hysteresis shifts of SCA, SEVA-C and SPCL were primarily caused by changes in the advancing arms of DCA force loops (Fig. 3). The concentration effect was more pronounced for SEVA-C and SPCL than for SCA. Moreover, SCA hysteresis approached a steady state close to zero during the initial 10 cycles. It was determined that higher concentrations did not yield different or further information, which could result from the formation of a monolayer. For SEVA-C and SPCL, the equilibrium between proteins in solution and those on the surface layer was observed around loop 30.

Despite the lower hydrophilicity of SEVA-C and SPCL than that of SCA, the hysteresis was affected by the protein solution and changed from a thermodynamic to a kinetic type. According to the literature, a cause of kinetic hysteresis is the adsorption of macromolecules from the liquid phase., ${ }^{4,16,17}$ When compared with the surface state before adsorption, the initial heterogeneity
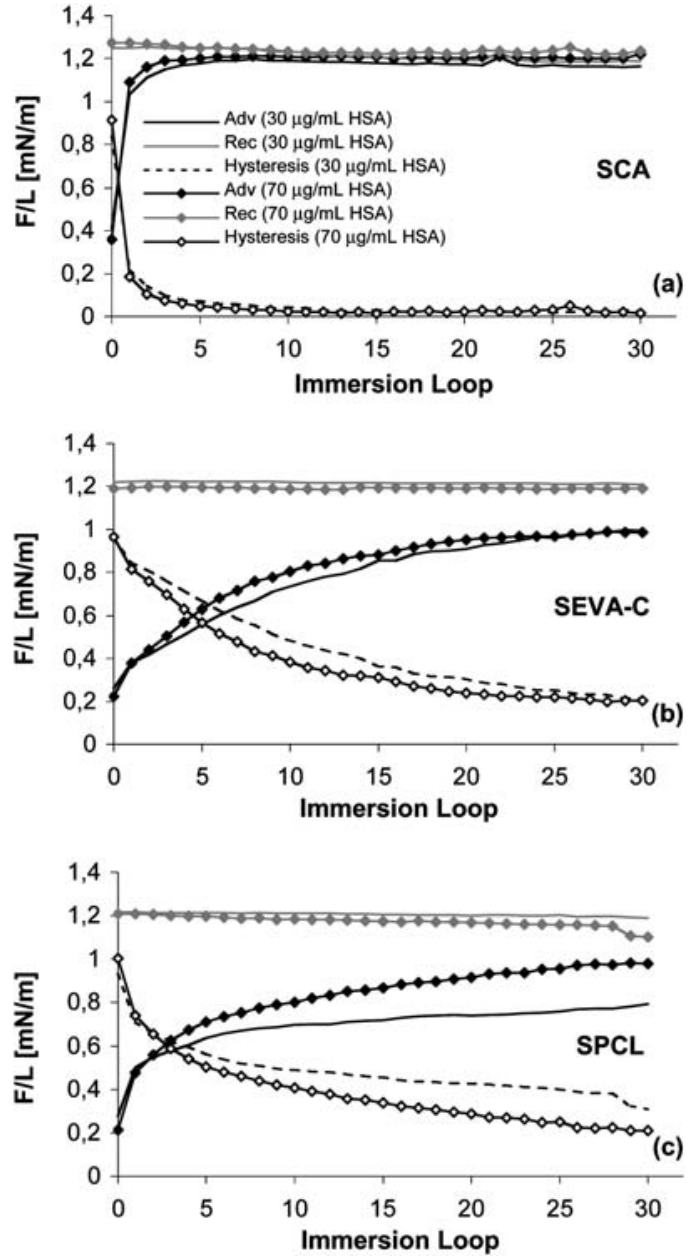

Fig. 3 30-loop DCA experiments showing hysteresis, advancing and receding wetting tensions obtained for $35 \mu \mathrm{g} \mathrm{mL}^{-1}$ and $70 \mu \mathrm{g} \mathrm{mL}{ }^{-1} \mathrm{HSA}$ solutions. SCA (a), SEVA-C (b) and SPCL (c) surfaces were analysed.

of a surface is affected by the onset of protein-surface interactions and kinetic hysteresis is a consequence of adsorption..$^{4,21}$ For all materials the DCA experiments in PBS contrasted with DCA studies in albumin solution (Fig. 1 and 2). Time dependent shifts in immersing and/or emerging forces have been demonstrated. Due to protein adsorption, the increase in hydrophilicity and the homogenization of the surfaces was demonstrated. Proteins were able to undergo conformational rearrangement to adsorb on both hydrophobic and hydrophilic surfaces. The hydrophilicity and decrease in hysteresis showed that the 

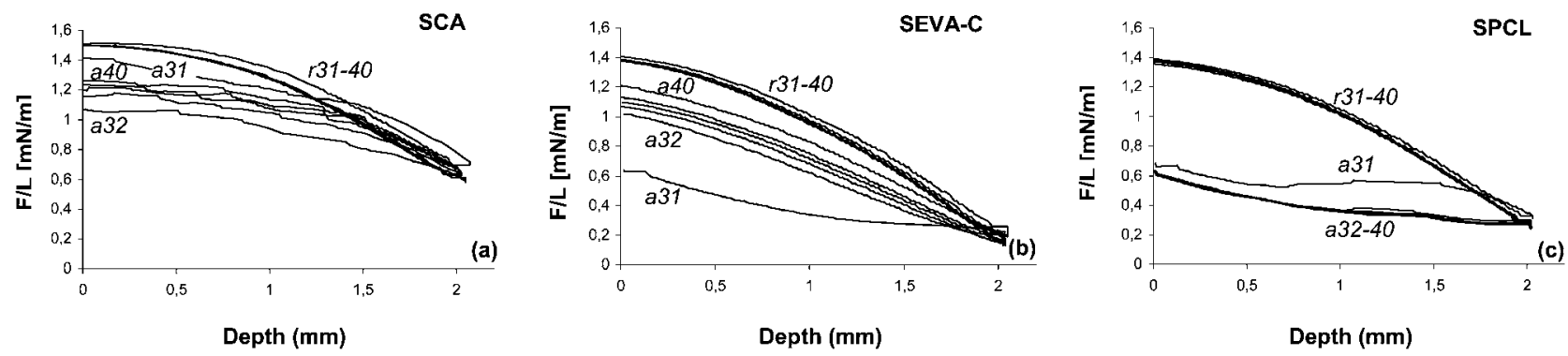

Fig. 4 DCA loops of SCA (a), SEVA-C (b) and SPCL (c) immersed in PBS solution (desorption phase) subsequently to the HSA adsorption experiment. The advancing $(a)$ and receding $(r)$ loop numbers (31-35 and 40) were indicated.

adsorption of albumin reduced the hydrophobic areas of the surface. In the present study, an energetically favourable system resulted from the rearrangement of the protein structure that exposed the hydrophilic regions towards the solution.

\subsection{Hysteresis of protein adsorption and desorption}

3.3.1. Single protein solutions. The adsorption/desorption studies were performed to analyse the reversibility of the wettability status, which may have changed during adsorption. Desorption studies were performed by replacing the solution of albumin, fibronectin, vitronectin and fibrinogen (adsorption phase) with PBS (Fig. 4). DCA results for SCA and SPCL surfaces demonstrated that the advancing arms of the contact angle loops irreversibly changed towards an increase in hysteresis. Similar trends were obtained for SEVA-C surfaces during the first cycles of the desorption phase. Nevertheless, at the end of the study, SEVA-C hysteresis returned to the levels observed for the adsorption phase (Fig. 2b and 4b).

The results of the adsorption/desorption experiments from the 60-loop Wilhelmy measurements using HSA are shown in Fig. 5. The equilibrium wetting tensions at loop 60 of the adsorption
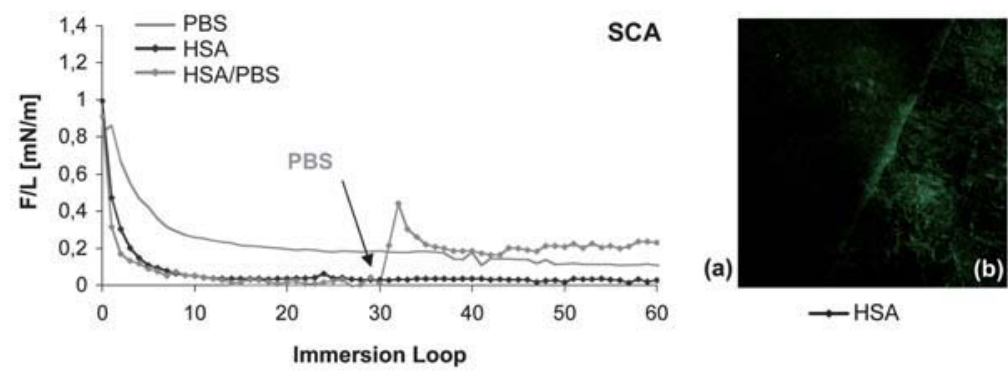

$\rightarrow$ HSA
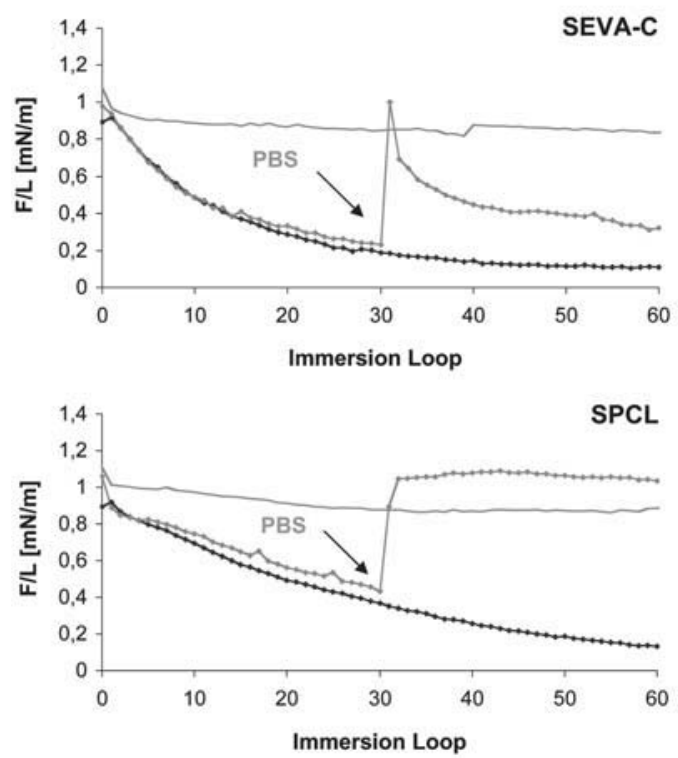

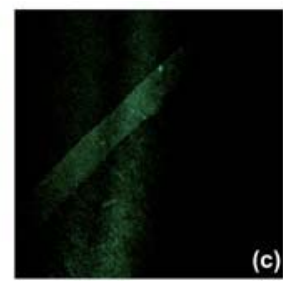

$\rightarrow$ HSA/PBS

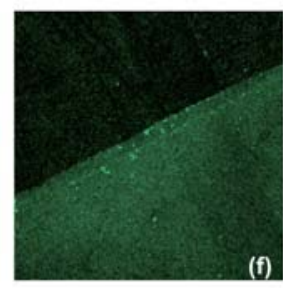

$\rightarrow$ HSAIPBS

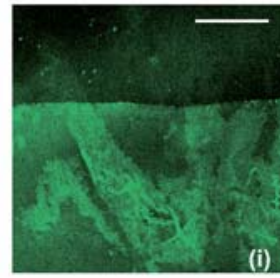

$\rightarrow$ HSA

Fig. 5 60-loop DCA adsorption/desorption hysteresis of SCA (a), SEVA-C (b) and SPCL (c) surfaces using $70 \mu \mathrm{g} \mathrm{mL}^{-1}$ HSA solutions. Adsorbed albumin at loop 30 (b, e and h) and at loop 60 (c, f and i) can be observed. Scale bar: $500 \mu \mathrm{m}$. 
study were nearly identical for SCA, SEVA-C and SPCL. In the study of protein desorption (loops 31-60), drastic changes could be observed and the influence of surface identity on the desorption kinetics was clear. Conditioning of SCA and SPCL with PBS during loops 31-60 showed a kinetic enhancing effect. The advancing and receding wetting tensions showed an immediate increase in hysteresis at the 31st loop, the first loop of the phase of desorption (Fig. 4b). The advancing data partially shifted back in the hydrophobic direction, whereas the receding wetting tension showed an irreversible behaviour, the original small hysteresis increased irreversibly. According to these DCA results, the adsorption of HSA on SPCL and SCA was reversible. In contrast, in the desorption experiments using protein-free solution showed that the hysteresis loop shift from the adsorption phase was irreversible on the SEVA-C surfaces. As for SCA and SEVA-C, the advancing wetting tension moved in the hydrophobic direction. However at loop 60, hysteresis values were similar to those obtained at the end of the adsorption experiment. Therefore, it can be concluded that HSA adsorption on SEVA-C was irreversible.

DCA experiments were also performed using HFn, HVn and $\mathrm{HFbg}$ at much lower concentrations. The results for SEVA-C are shown in Fig. 6. The trends observed for fibronectin, vitronectin and fibrinogen were similar to that of albumin, including a fast increase in hysteresis at loop 31. Also, at loop 60 the adsorption profiles were partially recovered for $\mathrm{HVn}$ and $\mathrm{HFbg}$, and totally recovered in the case of HFn. This showed that, in approximately $90 \mathrm{~min}$ (loop 31-60), the proteins adsorbed on SEVA-C rearranged their conformation back to the structure adopted during the adsorption stage (loop 1-30).

DCA was also performed on the surface of SCA and SPCL using HFn, HVn and HFbg test solutions. Yet, in adsorption and desorption experiments no changes in hysteresis were observed (Fig. 7a). Although protein adsorption was not detected by DCA analysis, it was observed by confocal microscopy performed after the adsorption (Fig. 7b) and desorption studies (Fig. 7c).

3.3.2. Competitive protein systems. Single protein solutions are generally used over complex protein mixtures for experimental simplicity. In the present study complex protein solutions were analysed in order to more closely evaluate the in vivo biological environment. To analyse the effect of protein competition on hysteresis, the data of 60 loop experiments was represented as a function of loop number (Fig. 8).

The adsorption and desorption experiments performed with human blood plasma strongly resembled those of HSA. The hysteresis curves and the hydrophobic shift obtained at loop 31 were very similar to the albumin single test solutions. In complex
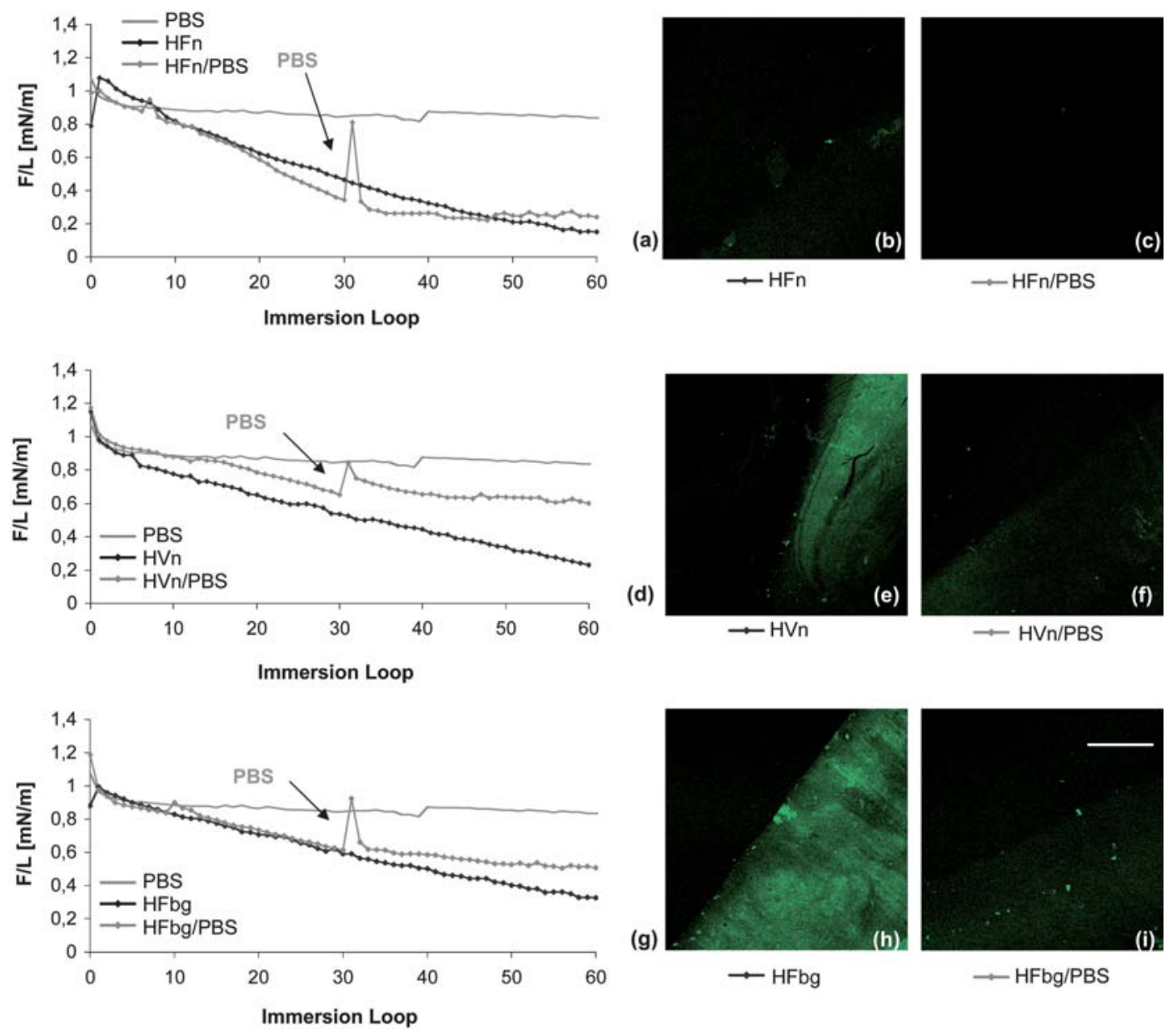

Fig. 6 60-loop DCA adsorption/desorption hysteresis of SEVA-C surfaces using $0.8 \mu \mathrm{g} \mathrm{mL}^{-1} \mathrm{HFn}$ (a), $0.6 \mu \mathrm{g} \mathrm{mL}-\mathrm{HVn}^{-1}$ (b) and $0.4 \mu \mathrm{g} \mathrm{mL}-1 \mathrm{HFbg}^{-(c)}$ solutions; and protein visualization at loop 30 (b, e and h) and at loop 60 (c, f and i). Scale bar: $500 \mu \mathrm{m}$. 


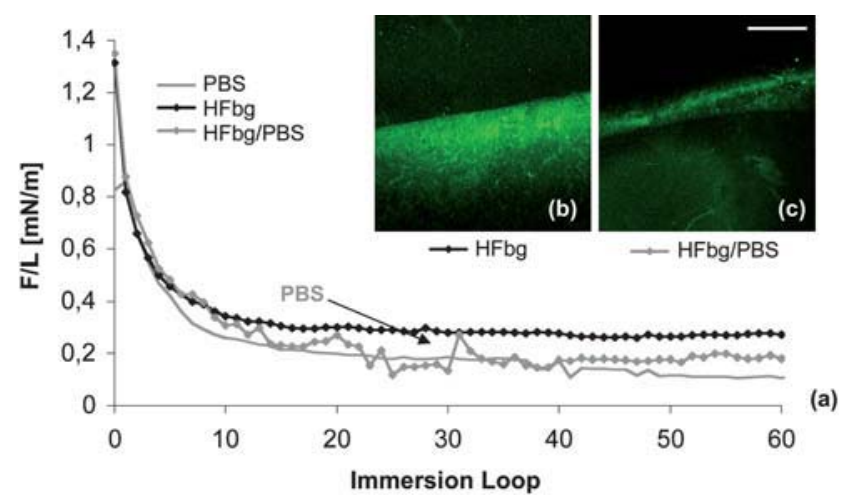

Fig. 7 60-loop DCA adsorption/desorption hysteresis of SPCL surfaces using PBS and $0.4 \mu \mathrm{g} \mathrm{mL}^{-1} \mathrm{HFbg}$ solutions (a) and protein visualization at loop 30 (b) and at loop 60 (c). Scale bar: $500 \mu \mathrm{m}$.

protein solutions, SCA and SPCL showed a hysteresis of zero before loop 10. In contrast only at loop 20, SEVA-C surfaces achieved complete wettability. Furthermore, SCA and SPCL desorption hysteresis did not recover to the original values obtained during the adsorption study (Fig. 8a and 8c). In contrast, SEVA-C achieved a completely wettable surface at both the end of the adsorption and desorption phases, at loop 30 and loop 60 respectively (Fig. 8b). This was not demonstrated for unitary HSA solutions (Fig. 5d). The increase in hydrophilicity of all the surfaces was higher for the competitive system due to the protein concentration and species diversity in the human blood plasma solution.

Changes in DCA hysteresis profiles were demonstrated to be related to material properties, although changes in hysteresis with HFn, HVn and HFbg solutions were not detected on SCA and SPCL and adsorption and desorption profiles obtained for SEVA-C were similar for the different molecules. In addition, SEVA-C showed no reversibility of the advancing wetting tensions and consequently, no changes in hysteresis. The results suggested that SEVA-C presented irreversible adsorption.

It is known that the wettability of a polymer affects protein adsorption and that hydrophobic surfaces tend to denature adsorbed proteins more than hydrophilic surfaces. ${ }^{34}$ Surface hydrophobicity strongly affects the interactions of proteins with materials and thus, the irreversibility of the adsorption process. When hydrophilic proteins adsorb onto hydrophilic surfaces no net hydrophobic interactions occur; additionally, no significant conformational changes take place in the tertiary and/or secondary structure of the protein. ${ }^{35,36}$ In previous studies,
SEVA-C was determined to be the most hydrophobic surface and, in contrast, SCA the most hydrophilic. ${ }^{37}$ It was previously demonstrated that the adsorption of proteins to a hydrophobic surface is usually irreversible due to the increase in the free energy gain, ${ }^{38,39}$ whereas the adsorption onto hydrophilic surfaces is weaker, more sensitive to electrostatic interactions and more reversible. ${ }^{40,41}$ Hydrophobic, electrostatic and specific acceptor donor interactions are generally referred to as the most important forces involved in the adsorption process. The surface charge plays a key role in the early electrostatic attraction of the protein to the surface. Nevertheless, these are relatively long distance forces. In contrast, hydrophobic interactions involve the release of water from both hydrophobic protein residues and the solid surface, which results in strong binding of the non-polar protein components with the surface. An irreversibly adsorbed protein layer is formed. Several other reversibly bound layers will then form on top of the irreversibly bound layer ${ }^{42}$ to create the complex protein multilayers which mediate cell interaction.

The differences of adhesion strength observed in the desorption phase that can be considered an elution test indicated conformational changes of the proteins. The surface mediated structure that was adopted by the proteins could affect the biological activity of the proteins and modulate the cell biological response to the different SBB.

\section{Conclusions}

The biological environment was simulated to determine single and competitive adsorption profiles of proteins found in blood onto the surface of materials. Insights into the dynamic equilibria established between proteins and surfaces were obtained from DCA measurements. Hysteresis profiles obtained for the different protein solutions indicated that different interactions between these proteins and substrates were taking place during the adsorption process. The SCA surface was the most interactive in terms of albumin adsorption, revealing saturation at shorter time periods as observed by the higher hydrophilicity. Adsorption studies with SEVA-C and SPCL showed very similar results and were in contrast to SCA.

The most significant differences were observed in the study of protein desorption. The differences observed in desorption indicated conformational changes of the proteins. SCA and SPCL were demonstrated to completely revert the hysteresis profile back to the original one obtained with protein-free buffer. In contrast, SEVA-C showed irreversible hysteresis independently of the protein used in the desorption study. The same results were
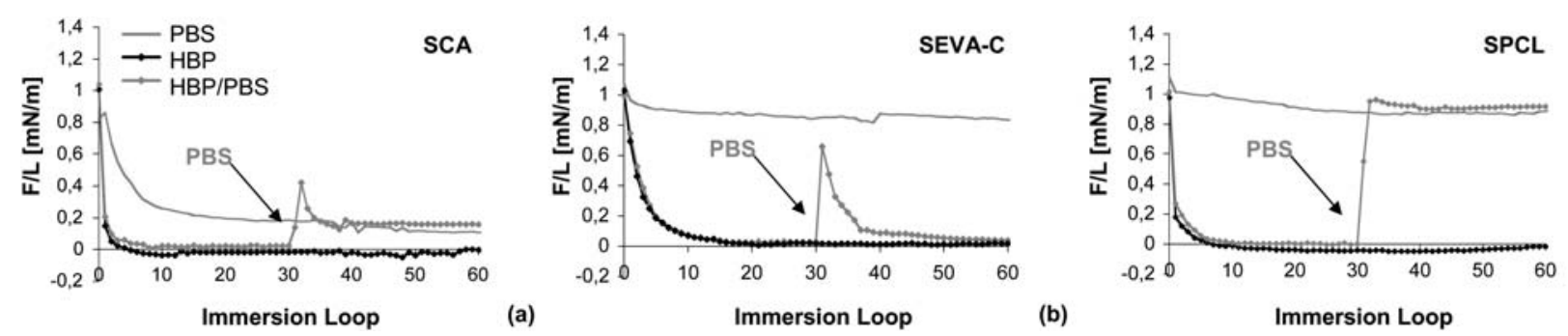

(c)

Fig. 8 60-loop DCA adsorption/desorption hysteresis of SCA (a), SEVA-C (b) and SPCL (c) surfaces using human blood plasma (HBP) solutions at $0.2 \%(\mathrm{v} / \mathrm{v})$. 
obtained for the study of protein competition. The biological activity of the proteins on the SBB surfaces was affected by the material properties. This study elucidated further the mechanisms of protein adsorption and the strength of the bonds formed before and after the onset of hysteresis. These results were in agreement with previous cell studies and could therefore explain the biological response to the different starch-based materials.

\section{Acknowledgements}

The authors acknowledge funding from the Foundation for Science and Technology (FCT), Portugal for SFRH/BD/11188/ 2002 and for partial funding through FEDER and POCTI programmes and EU funded Project HIPPOCRATES (NMP3-CT2003-505758). This work was carried out under the scope of the European Network of Excellence EXPERTISSUES (NMP3-CT2004-500283).

\section{References}

$1 \mathrm{~J}$. L. Brash, Exploiting the current paradigm of blood-material interactions for the rational design of blood-compatible materials, J. Biomater. Sci., Polym. Ed., 2000, 11(11), 1135-46.

2 J. Nikolovski and D. J. Mooney, Smooth muscle cell adhesion to tissue engineering scaffolds, Biomaterials, 2000, 21(20), 2025.

3 C. J. Wilson, R. E. Clegg, D. I. Leavesley and M. J. Pearcy, Mediation of biomaterial-cell interactions by adsorbed proteins: a review, Tissue Eng., 2005, 11(1-2), 1-18.

4 J. D. Andrade; L. M. Smith; D. E. Gregonis, The contact angle and interface energetics. In Surface and Interfacial Aspects of Biomedical Polymers, ed. J. D. Andrade., Plenum: New York, 1985; Vol. 1, pp 249-292.

5 C. H. Thomas, C. D. McFarland, M. L. Jenkins, A. Rezania, J. G. Steele and K. E. Healy, The role of vitronectin in the attachment and spatial distribution of bone-derived cells on materials with patterned surface chemistry, J. Biomed. Mater. Res., 1997, 37(1), 81-93.

6 J. L. Guan, J. E. Trevithick and R. O. Hynes, Fibronectin Integrin Interaction Induces Tyrosine Phosphorylation of a $120-\mathrm{Kda}$ Protein, Cell Regul., 1991, 2(11), 951-964.

7 J. L. Yu, S. Johansson and A. Ljungh, Fibronectin exposes different domains after adsorption to a heparinized and an unheparinized poly(vinyl chloride) surface, Biomaterials, 1997, 18(5), 421-7.

8 D. G. Castner and B. D. Ratner, Biomedical surface science: Foundations to frontiers, Surf. Sci., 2002, 500(1-3), 28-60.

9 F. Y. Liu, M. Y. Zhou and F. Zhang, I-125 labelling of human serum albumin and fibrinogen and a study of protein adsorption properties on the surface of titanium oxide film, Appl. Radiat. Isot., 1998, 49(12), 67-72.

10 A. Kondo and H. Fukuda, Effects of Adsorption Conditions on Kinetics of Protein Adsorption and Conformational Changes at Ultrafine Silica Particles, J. Colloid Interface Sci., 1998, 198(1), 34.

11 T. Ueda, K. Ishihara and N. Nakabayashi, Adsorption-desorption of proteins on phospholipid polymer surfaces evaluated by dynamic contact angle measurement, J. Biomed. Mater. Res., 1995, 29(3), $381-7$.

12 E. A. Vogler, Structure and reactivity of water at biomaterial surfaces, Adv. Colloid Interface Sci., 1998, 74(1-3), 69-117.

13 H. Stadler, M. Mondon and C. Ziegler, Protein adsorption on surfaces: dynamic contact-angle (DCA) and quartz-crystal microbalance (QCM) measurements, Anal. Bioanal. Chem., 2003, 375(1), 53-61.

14 J. D. Whittle, N. A. Bullett, R. D. Short, C. W. I. Douglas, A. P. Hollander and J. Davies, Adsorption of vitronectin, collagen and immunoglobulin-G to plasma polymer surfaces by enzyme linked immunosorbent assay (ELISA), J. Mater. Chem., 2002, 12, 2726-2732.

15 L. Mhamdi, C. Picart, C. Lagneau, A. Othmane, B. Grosgogeat, N. Jaffrezic-Renault and L. Ponsonnet, Study of the polyelectrolyte multilayer thin films' properties and correlation with the behavior of the human gingival fibroblasts, Mater. Sci. Eng., C, 2006, 26(23), 273 .

16 R. E. Johnson and R. Dettre, Wettability and contact angles, Surf. Colloid Sci., 1969, 2, 85-153.

17 F. Rupp, L. Scheideler and J. Geis-Gerstorfer, Effect of heterogeneic surfaces on contact angle hyteresis: dynamic contact angle analysis in materials science, Chem. Eng. Technol., 2002, 25(9), 877-881.

18 M. Miyama, Y. Yang, T. Yasuda, T. Okuno and H. K. Yasuda, Static and Dynamic Contact Angles of Water on Polymeric Surfaces, Langmuir, 1997, 13, 5494-5503.

19 F. Rupp, D. Axmann, C. Ziegler and J. Geis-Gerstorfer, Adsorption/ desorption phenomena on pure and Teflon AF-coated titania surfaces studied by dynamic contact angle analysis, J. Biomed. Mater. Res., 2002, 62(4), 567-578.

$20 \mathrm{M}$. Mondon and C. Ziegler, Changes in water contact angles during the first phase of setting of dental impression materials, Int. J. Prosthodont., 2003, 16(1), 49-53.

21 H. Stadler, M. Mondon and C. Ziegler, Protein adsorption on surfaces: dynamic contact-angle (DCA) and quartz-crystal microbalance (QCM) measurements, Anal. Bioanal. Chem., 2003, 375(1), 53-61.

22 M. E. Gomes, J. S. Godinho, D. Tchalamov, A. M. Cunha and R. L. Reis, Alternative tissue engineering scaffolds based on starch: processing methodologies, morphology, degradation and mechanical properties, Mater. Sci. Eng., C, 2002, 20(1-2), 19-26.

23 A. J. Salgado, O. P. Coutinho and R. L. Reis, Novel starch-based scaffolds for bone tissue engineering: Cytotoxicity, cell culture, and protein expression, Tissue Eng., 2004, 10(3-4), 465-474.

24 A. L. Oliveira, C. M. Alves and R. L. Reis, Cell adhesion and proliferation on biomimetic calcium-phosphate coatings produced by a sodium silicate gel methodology, J. Mater. Sci.: Mater. Med., 2002, 13(12), 1181-1188.

25 C. Elvira, J. F. Mano, J. San Roman and R. L. Reis, Starch-based biodegradable hydrogels with potential biomedical applications as drug delivery systems, Biomaterials, 2002, 23(9), 1955-1966.

26 A. P. Marques, R. L. Reis and J. A. Hunt, The effect of starch-based biomaterials on leukocyte adhesion and activation in vitro, J. Mater. Sci.: Mater. Med., 2005, 16(11), 1029-43.

27 R. L. Reis, S. C. Mendes, A. M. Cunha and M. J. Bevis, Processing and in vitro degradation of starch/EVOH thermoplastic blends, Polym. Int., 1997, 43(4), 347-352.

28 J. Wilhelmy, Uber die Abhandigkeit der Capilaritats-Constanten des alkohols von Substanz und Gestalt des benetzten festen Kopers, Ann. Phys., 1863, 119, 177-217.

29 C. M. Alves, R. L. Reis and J. A. Hunt, The competitive adsorption of human proteins onto natural-based biomaterials, J. R. Soc. Interface, 2010, DOI: 10.1098/rsif.2010.0022.

30 C. M. Alves, R. L. Reis and J. A. Hunt, Preliminary Study on Human Protein Adsorption and Blood Cells Adhesion to Starch-Based Biomaterials, J. Mater. Sci.: Mater. Med., 2003, 14, 157-165.

31 I. Pashkuleva, H. S. Azevedo and R. L. Reis, Surface Structural Investigation of Starch-Based Biomaterials, Macromol. Biosci., 2008, 8, 210-219.

32 R. E. Johnson and R. H. Dettre, Contact Angle Hysteresis. III. Study of an idealized heterogeneous surface, J. Phys. Chem., 1964, 68, 17441750.

33 R. H. Dettre and R. E. Johnson, Contact Angle Hysteresis. IV. Contact angle measurements on heterogeneous surface, J. Phys. Chem., 1965, 69, 1507-1516.

34 T. A. Horbett and A. S. Hoffman, Bovine Plasma-Protein Adsorption onto Radiation-Grafted Hydrogels Based on Hydroxyethyl Methacrylate and N-Vinyl-Pyrrolidone, Adv. Chem. Ser., 1975, 145, 230-254.

35 J. O. Naim, C. J. van Oss, K. M. L. Ippolito, J.-W. Zhang, L.-P. Jin, R. Fortuna and N. A. Buehner, In vitro activation of human monocytes by silicones, Colloids Surf., B, 1998, 11(1-2), 79-86.

36 C. J. van Oss, A. Docoslis, J. Giese and F. Rossman, Free energies of protein adsorption onto mineral particles - from the initial encounter to the onset of hysteresis, Colloids Surf., B, 2001, 22(4), 285-300.

37 C. M. Alves, Y. Yang, D. L. Carnes, J. L. Ong, V. L. Sylvia, D. D. Dean, C. M. Agrawal and R. L. Reis, Modulating bone cells response onto starch-based biomaterials by surface plasma treatment and protein adsorption, Biomaterials, 2007, 28(2), 307.

38 J. D. Andrade, V. L. Hlady and R. A. Vanwagenen, Effects of Plasma-Protein Adsorption on Protein Conformation and Activity, Pure Appl. Chem., 1984, 56(10), 1345-1350. 
39 E. J. Castillo, J. L. Koenig, J. M. Anderson and J. Lo, Protein adsorption on hydrogels: II. Reversible and irreversible interactions between lysozyme and soft contact lens surfaces, Biomaterials, 1985, 6(5), 338-345.

40 U. Jonsson, B. Ivarsson, I. Lundstrom and L. Berghem, Adsorption behavior of fibronectin on well-characterized silica surfaces, J. Colloid Interface Sci., 1982, 90(1), 148.
41 U. Jonsson, I. Lundstrom and I. Ronnberg, Immunoglobulin G and secretory fibronectin adsorption to silica: The influence of conformational changes on the surface, J. Colloid Interface Sci., 1987, 117(1), 127.

42 T. P. Burghardt and D. Axelrod, Total internal reflection/fluorescence photobleaching recovery study of serum albumin adsorption dynamics, Biophys. J., 1981, 33(3), 455-67. 